\title{
ÉDITORIAL
}

\section{Bien-être et performance : quel avenir?}

Devant l'inéluctable marche vers l'avenir de l'humain au travail, il faut savoir apprécier le chemin parcouru et prendre le temps d'observer l'évolution du statut de salariat au cours du vingtième siècle. La progression du droit statutaire a permis de construire un régime formel de conditions de travail nécessaire au développement d'une nouvelle philosophie axée sur le mieux-être des salariés. Après avoir notamment obtenu une protection d'emploi, le droit d'association et la régulation des heures travaillées, la société du travail entre dans une ère où le présent côtoie l'avenir dans le déploiement d'une vision partagée du travail afin d'assurer la croissance et la pérennité des entreprises. Ce constat doit être apprécié au regard des efforts déployés par les organisations pour mieux encadrer "l'expérience de travail » du collectif salarié. L'étude des milieux organisationnels permet justement de distinguer des thèmes probants en relation directe avec le management. À ce titre, l'expérience positive des salariés peut être liée à l'aménagement de conditions de travail permettant l'épanouissement, la conciliation du travail et de la vie personnelle ou tout autre nouvelle forme d'organisation du travail. Les résultats de ces efforts peuvent toutefois être remise en question par la présence d'un paradigme de performance qui peut avoir comme conséquence la réduction de la portée des actions concertées misant sur le mieux-être des salariés.

Malgré les gains réalisés par les salariés au niveau de leurs conditions et de la qualité de l'environnement de travail, la recherche incessante de la performance à tout prix peut obscurcir l'arc-en-ciel managérial déployé. Puisque la responsabilité et l'imputabilité se caractérisent comme des éléments centraux des nouvelles formes de management, on peut dès lors observer une dichotomie managériale entre l'intensification de la charge de travail et le bien-être des salariés. Une question se pose alors, à savoir quel sera le prix à payer pour l'humain au travail. Certains constats se dressent déjà en regard de la rareté de la main-d'œuvre, des problèmes de santé mentale ou des difficultés à développer un contexte mobilisant. La société du travail actuel vacille donc sur un équilibre précaire entre le bien-être des salariés et la performance des organisations.

Dans le cadre de ce numéro intitulé « Avenir, bien-être et performance », nous avons la chance d'approfondir nos réflexions grâce à plusieurs contributions de chercheurs intéressés à la santé des travailleurs, au rôle des gestionnaires, au développement d'une identité organisationnelle et, bien entendu, à la performance organisationnelle. Il faut souligner les efforts déployés dès le début du projet, par tous les auteurs pour faire de la revue Ad machina un incontournable pour le milieu universitaire. Par la diffusion des résultats de leurs activités de recherche, la publication du présent numéro nous permet d'affirmer qu'Ad machina occupe désormais une place de choix dans les revues francophones en libre accès sur le travail. Nous espérons que vous prendrez plaisir à la lecture de ce numéro.

Le comité éditorial,

Olivier Gagnon, Université du Québec à Chicoutimi

Éric Jean, Université du Québec à Chicoutimi

Stéphanie Maillet, Université de Moncton

Doi: 10.1522/radm.no3.1094 